\title{
Skewed demographics
}

\section{Scientists based in North America and men are overrepresented in our authors' reviewer suggestions.}

Invitation-only activities can be a mixed blessing, and peer review is no exception. Assessing fellow scientists' manuscripts is an important part of a scientific career with numerous professional benefits, but on the negative side, conscientious peer review can eat up copious amounts of time. Thus, in an ideal world, peer review would be distributed evenly across the relevant scientific community.

To understand our own biases at Nature Geoscience, as well those of our community, we collected data on gender and geographic location of our authors, and their reviewer recommendations for a four-month period (13 January to 12 May 2017). Not entirely unexpectedly, reviewer recommendations are biased - compared to author demographics - towards men and those at North American institutions, the latter at the expense of researchers in Asia. Our assignments of reviewers within the editorial team achieve a better gender balance than our authors' suggestions. In terms of geography, however, similar biases exist.

In terms of geography (Fig. 1a), of the 676 corresponding authors who submitted during the four months of data collection, Europe/ Middle East was the most frequent affiliation (39\%), followed by North America (33\%) and Asia (23\%). Researchers at European or Middle Eastern institutions were suggested as reviewers roughly according to author proportion (36\%). However, the share of North Americans was much higher (54\%), whereas scientists at Asian institutions were recommended much less often than their share in authorship would imply (6\%).

Looking at only the subset of authors whose papers were sent out for review (about $20 \%$ of the papers received, and arguably a more justifiable baseline), authorship proportions are somewhat different. Europe and the Middle East are similarly represented (41\%), but North American affiliations are found more often in the subset (39\%), and Asian affiliations less frequently (15\%). Despite these differences in the author demographics, reviewer suggestions from the subset follow broadly similar proportions as suggestions from all authors.

At Nature Geoscience, reviewer suggestions from authors have only a small influence: as editors, we mostly assemble reviewer panels independent from authors' suggestions, by matching prospective reviewers' publications
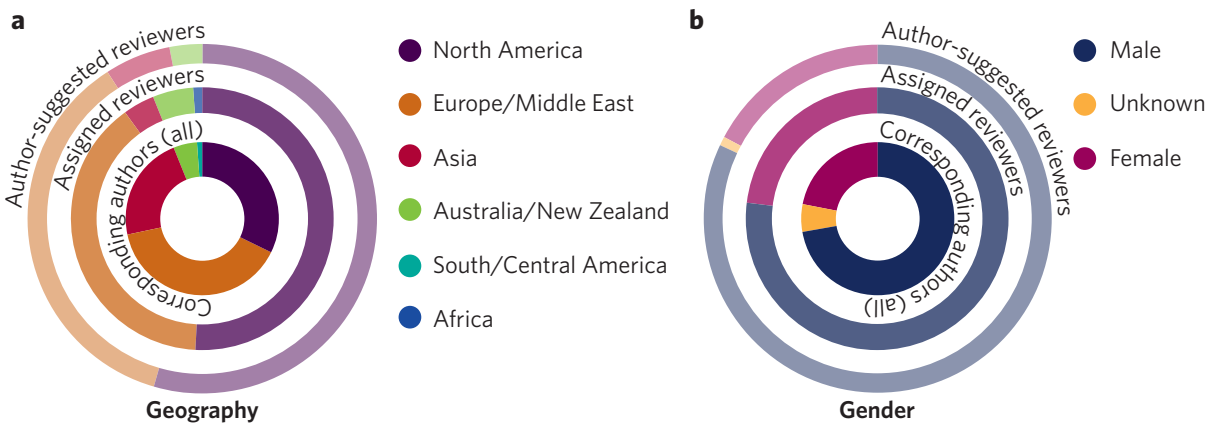

Figure 1 | Gender and geography distributions of authors and reviewers.

and research interests to each paper. Indeed, we aim to assign no more than one authorsuggested reviewer per paper in order to ensure rigorous peer review; for example, for the field of ecology, a positive bias has been documented in the assessments by author-suggested reviewers ${ }^{1}$. Nevertheless, the geographical distribution of editor-assigned reviewers resembles the biases of authorsuggested reviewers (Fig. 1a).

In light of this survey, we will endeavour to even out geographical imbalances and seek out more potential reviewers from Asia. This is a good time to start: as of September, our editor Rebecca Neely will be based in Shanghai. She will explore the Asian research landscape and share recommendations with editors based elsewhere. We would also appeal to researchers at Asian institutions to improve their internet presence.

As a second focus of this survey, we collected data on gender. Of all of the corresponding authors, $72 \%$ were men, $22 \%$ women, and for $6 \%$ we were unable to determine gender (marked as 'unknown'). Among recommended reviewers, however, the proportion of women was substantially lower (17\%, Fig. 1b). For the subset of manuscripts selected for peer review, $72 \%$ of authors were male - comparable to the proportion among all authors. However, a larger fraction (27\%) were female, with only $1 \%$ unknown. If authors of unknown gender roughly follow the 3:1 male/female proportions of all authors, women as corresponding authors are slightly more successful in having their papers sent out for review than men. Such a finding would be consistent with an assessment of author demographics by the American Geophysical
Union (AGU) ${ }^{2}$, which found a higher proportion of female first authors among published than submitted papers. However, if most unknown-gender authors are female then the differences are insignificant.

Also in line with demographics at the AGU publications ${ }^{2}$, women suggested a higher proportion of female reviewers (20\%) than men (16\%). In contrast to our findings on geography, Nature Geoscience editors chose reviewers that reflect author demographics more closely than author-suggested reviewers. The data we collected and this analysis will, although it only scratches the surface of the complexities of bias in gender and geography, provide much food for thought for us. It will also help us to develop strategies to mitigate the editorial imbalances we identified. We are sharply aware that it is in the interest of publishing the best scientific papers - and hence our interest - to enlist a diverse pool of talented scientists in peer review.

But a change in culture must be a community effort, too. A stunning two-fifths of papers with reviewer recommendations suggested an all-male panel. On only two papers was the panel all female. So here is a challenge for our authors (particularly those based in Europe and North America): come up with at least one suggested reviewer based outside of Europe, North America, Australia or New Zealand, and at least one woman, on each paper that you submit next year.

\footnotetext{
References

1. Fox, C. W., Burns, C. S., Muncy, A. D. \& Meyer, J. A. Funct. Ecol. 31, 270-280 (2017).

2. Lerback, J. \& B. Hanson, B. Nature 541, 455-457 (2017).
}

Additional information

Supplementary information is available in the online version of this paper. 